\title{
COVID-19 Related Daily Stressors, Coping, and Suicidal Ideation in Psychiatrically Hospitalized Youth
}

\author{
Anastacia Y. Kudinova ${ }^{1,2}$ (D) . Alexandra H. Bettis ${ }^{3}$. Elizabeth C. Thompson ${ }^{1,4}$. \\ Sarah A. Thomas ${ }^{1,4}$. Jacqueline Nesi ${ }^{1,4}$. Leyla Erguder $^{1,2} \cdot$ Heather A. MacPherson $^{1,2}$. \\ Taylor A. Burke ${ }^{1,4}$. Jennifer C. Wolff ${ }^{1,2}$
}

Accepted: 2 July 2021 / Published online: 19 July 2021

(c) The Author(s), under exclusive licence to Springer Science+Business Media, LLC, part of Springer Nature 2021

\begin{abstract}
Background Given reports of the adverse effects of COVID-19 on adolescent mental health, it is critical to understand how it impacts psychiatrically hospitalized youth who may be particularly vulnerable to its effects.

Objective This study aimed to advance our understanding of high-risk adolescents' experiences of COVID-19, including COVID-19-related stress, changes in daily functioning, and coping as they relate to suicidal ideation (SI).

Method Participants were 107 youth (ages 11-18; $\mathrm{M}=15.06, S D=1.79$ ) admitted to an adolescent psychiatric inpatient unit during the time when the initial COVID-19 safety measures (i.e., school closure, stay-at-home- order) and reopening initiatives (Phase I, II, and III) were implemented in Rhode Island between March 13th and July 19th 2020. Adolescents completed measures of COVID-19-related stress, coping, functioning, and SI at the time of admission.

Results Nearly half of the sample (43\%) reported a negative impact of COVID-19 on daily functioning. Youth who endorsed COVID-19-related decline in functioning evidenced higher levels of SI compared to youth with no change or improvement in functioning due to COVID-19. Overall levels of stress were not associated with SI. Greater coping repertoire, but not the use of specific coping strategies was associated with higher levels of SI.

Conclusions Findings demonstrate the importance of examining COVID-19-related changes in functioning and broadening repertoire of coping strategies among adolescents at high risk for SI.
\end{abstract}

Keywords COVID-19 · Coping · Daily functioning $\cdot$ Stress · Psychiatrically hospitalized youth

AnastaciaY. Kudinova and AlexandraH. Bettis have contributed equally.

Anastacia Y. Kudinova

Anastacia_kudinova@brown.edu

Extended author information available on the last page of the article 


\section{Introduction}

The COVID-19 pandemic has created new and unique challenges, as well as exacerbated existing ones, for adolescents. Exposure to COVID-19-related stressors, including disruptions in daily functioning, may magnify negative mood and existing mental health problems in youth (Golberstein et al., 2019, 2020). Recent calls for COVID-19 research in adolescents highlight the importance of understanding how these changes are impacting youth mental health (Fegert et al., 2020; Gruber et al., 2020; Gunnell et al., 2020; Holmes et al., 2020). Youth with a history of mental health problems, such as those requiring psychiatric hospitalization, may be particularly vulnerable to the effects of the pandemic (Asmundson et al., 2020; Guessoum et al., 2020). The ways in which vulnerable youth cope with stress in the context of COVID-19 may mitigate or worsen the impact of pandemic-related stress on their psychological health, including experiencing suicidal ideation (SI). This study aims to advance our understanding of high-risk adolescents' experiences of the COVID-19 pandemic.

Exposure to stress is associated with increased negative mental health outcomes and exposure to acute stressful events is associated with elevated risk for SI in youth (Hammen, 2005; Liu \& Miller, 2014; O'Connor et al., 2010). The COVID-19 pandemic has amplified stress across life domains, including increased social isolation, disruptions in school and work, health-related concerns, and strain in interpersonal relationships and family finances. In a survey of 1,054 high school Canadian youth, over 75\% reported being "very" concerned about the pandemic's effects on school; nearly half of the sample reported worries about staying connected with friends; and 43\% reported feeling "very much" concerned about the pandemic overall (Ellis et al., 2020). For adolescents psychiatrically hospitalized during the pandemic, understanding COVID-19 specific stressors and their mental health correlates is critical to inform treatment targets. Importantly, as these youth have existing mental health problems, the onset of the COVID-19 pandemic and the associated stressors may have particularly detrimental consequences. The onset of the pandemic created an environment in which the stressors of everyday life were amplified simultaneously and rapidly; for example, schools closed and stay-at-home orders were put into place over a very brief period of time across the US, with little warning or time for preparation. Multiple acute stressors occurring at one time may have compounding effects on youth already vulnerable to mental health challenges, particularly stressors such as social isolation, disruptions in routines, and managing a significant amount of uncertainty about the immediate and long-term future.

To date, studies find the pandemic is associated with elevated distress and mental health problems in adolescents. In a sample of 3,613 Chinese youth, $91 \%$ reported concerns about COVID-19. Further, anxiety levels were significantly higher in youth compared to pre-pandemic, and approximately one quarter reported clinically significant depressive symptoms (Duan et al., 2020). Nearly $20 \%$ of adolescents in a community sample reported suicidal thoughts, and COVID-19 stress predicted elevated depressive symptoms in a survey of Canadian youth (Ellis et al., 2020). However, to our knowledge, no studies have examined COVID-19 related stress and subsequent changes in daily functioning in psychiatrically hospitalized adolescents prior to their admission, and whether their coping responses and available repertoire of coping skills may be linked to SI severity at intake.

Theoretical models of coping purport that how individuals cope with stress may be categorized into two broad factors, engagement and disengagement coping, and that these coping factors are associated with mental health outcomes (Compas et al., 2001, 2017). 
Empirical research testing this theory finds the use of engagement strategies (e.g., relaxation, social support, reappraisal, distraction) are associated with reduced internalizing and externalizing symptoms, whereas the use of disengagement strategies (e.g., avoidance, wishful thinking) are associated with worsening of these symptoms (Compas et al., 2017). Theoretical models of emotion regulation also emphasize the importance of flexibility in the use of strategies to manage stress (Bonanno \& Burton, 2013; Gross, 2015). Supporting the emotion regulation flexibility model, studies have found that having a larger repertoire of coping strategies and the ability to switch flexibly between multiple strategies has also been linked to resilient outcomes in various contexts (Cheng, 2001; Southward et al., 2018). Further, controllability is a central feature of theoretical models of coping with stress, and research has found evidence to suggest that some engagement strategies may be best suited for uncontrollable vs. controllable stressors (Rudolph, Dennig, \& Weisz, 1995; Weisz, McCabe, \& Dennig, 1994; Compas et al., 2012). Thus, how adolescents cope with pandemic-related stress may play a critical role in their psychological well-being during this time of significant uncertainty and uncontrollability (Polizzi et al., 2020). In a study of behavioral and emotional problems in Chinese children, results suggested the use of behavioral distractions such as the use of media, exercise, and reading as entertainment helped mitigate the negative impact of COVID-19 (Jiao et al., 2020). Research is needed to understand whether engagement strategies, as compared to disengagement strategies, are associated with less pandemic-related distress and SI among highly vulnerable youth.

Understanding which strategies youth report they are using to manage distress in the pandemic, what their coping repertoire is, and how they relate to suicide risk is especially important, given many avenues for using coping strategies have been limited by social distancing measures, school closure, and restrictions on large gatherings. A cumulative biological burden of adapting to rapidly changing and/or challenging circumstances often results in poor mental and physical health (Danese \& McEwen, 2012). For youth who experience periods of acute clinical distress including SI, increased levels of stress due to the pandemic in conjunction with reduced access to typical activities used to manage stress (i.e., the potential for a reduced coping repertoire) could elevate the risk for mental health problems, including SI.

\section{The Current Study}

Research describing the adverse impact of COVID-19 on youth mental health to date has largely focused on community samples (Singh et al., 2020), and no studies, to our knowledge, have examined these processes in youth at a high risk for suicide (Guessoum et al., 2020). Given the duration, widespread impact, and uncertain future of the pandemic, it is crucial to determine how adolescents vulnerable to serious mental illness are responding to and experiencing COVID-19. Thus, the current study aims to extend prior COVID-19 research in youth to understand the pandemic's impact in a psychiatrically hospitalized sample. Specifically, we aimed to characterize how youth retrospectively report experiencing the COVID-19 pandemic immediately prior to psychiatric hospitalization, with regard to COVID-19 related stress and impact on daily functioning, coping strategy use and repertoire, and SI at time of hospitalization. Three primary hypotheses were tested are: (1) Higher levels of COVID-19 related stress and greater COVID-19 related negative changes in functioning would be associated with greater SI severity. (2) Greater use of engagement coping strategies (i.e., social engagement, 
relaxation, distraction) and reduced use of disengagement coping strategies would be associated with lower SI (Mirkovic et al., 2020; Nrugham et al., 2012). (3) Larger coping repertoire would be linked lower levels of SI.

\section{Method}

\section{Sample and Procedures}

Participants were 107 youth, $11-18$ years old $(\mathrm{M}=15.06, S D=1.79)$, admitted to an adolescent psychiatric inpatient unit between March 13th and July 19th 2020, when the initial COVID-19 safety measures (e.g., transitioning to online learning at schools) and reopening steps were implemented in Rhode Island (Fig. 1). Reasons for admission to the inpatient unit include being at a risk of harming themselves or others. The sample included the following self-reported gender identities: $47.7 \%$ female, $43.9 \%$ male, $5.6 \%$ Gender-Queer/Gender Non-Conforming/Gender Fluid, and 1.9\% transgender male; $0.9 \%$ preferred not to answer. The majority of youth were non-Hispanic (66.4\%). Participants identified as White (53.2\%), Black or African American (13.0\%), American Indian or Alaska Native (1.9\%), of multiple races/ethnicities (16.8\%), and from other racial/ethnic backgrounds (14.0\%). As typical of an inpatient sample, a large proportion of youth had clinically significant levels of internalizing (43.9\%) and externalizing $(15.9 \%)$ symptoms and attention problems $(29.0 \%)$ at the time of admission.

Data were gathered during the standard intake process: Psychology staff administered self-report measures to all newly admitted patients to inform clinical care. The hospital's Institutional Review Board approved a waiver of informed consent for this chart review study.

\begin{tabular}{|c|c|c|c|c|c|c|c|}
\hline $\begin{array}{l}\text { COVID-19 } \\
\text { Milestones }\end{array}$ & $\begin{array}{l}\text { March } 13 \\
\text { Schools } \\
\text { transition } \\
\text { to online } \\
\text { learning }\end{array}$ & $\begin{array}{l}\text { March } 28 \\
\text { Stay-at- } \\
\text { home order } \\
\text { starts }^{2}\end{array}$ & $\begin{array}{l}\text { April } 2020 \\
\text { First peak of } \\
\text { new daily } \\
\text { COVID-19 } \\
\text { cases }^{3}\end{array}$ & $\begin{array}{c}\text { May } 9 \\
\text { Phase I } \\
\text { reopening } \\
\text { starts }^{4}\end{array}$ & $\begin{array}{c}\text { June } 1 \\
\text { Phase II } \\
\text { reopening } \\
\text { starts } 5\end{array}$ & $\begin{array}{c}\text { June } 30 \\
\text { Phase III } \\
\text { reopening } \\
\text { starts }^{6}\end{array}$ & \\
\hline $\begin{array}{l}\text { Month of } \\
2020\end{array}$ & $\mathrm{Mar}$ & arch & April & May & Ju & ne & July \\
\hline $\begin{array}{l}\text { Study } \\
\text { Enrollment } \\
\text { Milestones }\end{array}$ & $\begin{array}{l}\text { March } 13 \\
\text { Study } \\
\text { enrollment } \\
\text { begins }\end{array}$ & & \multicolumn{4}{|c|}{ Ongoing study enrollment } & $\begin{array}{c}\text { July } 19 \\
\text { Study } \\
\text { enrollment } \\
\text { ends }\end{array}$ \\
\hline
\end{tabular}

Fig. 1 Study enrollment timeline in relation to COVID-19 safety measures and reopening stages in Rhode Island. ( ${ }^{1}$ Schools transition to Online learning following the Governor's order for school closure, ${ }^{2}$ Stay-athome order is issued which instructs individuals to only leave homes to perform essential activities. The order also directs non-essential businesses to close. ${ }^{3} \mathrm{New}$ daily COVID-19 cases reach the highest numbers since the start of the pandemic, peaking at 36.9 per 100,000 . ${ }^{4}$ Phase I initiates limited re-opening of nonessential businesses under the safety requirements of face coverings and social distancing. Gatherings of up to 5 individuals are now allowed. ${ }^{5}$ Phase II initiates the opening of even more non-essential businesses with expanded limited capacities. Gatherings of up to 15 individuals are now allowed. ${ }^{6}$ Phase III furthers the opening of non-essential businesses. Gatherings of up to 25 individuals indoors and up to 50 individuals outdoors are now allowed. This figure is based on the timeline described in Thompson et al. (2021) study). 


\section{Measures}

\section{Suicidal Ideation}

The Suicidal Ideation Questionnaire-Junior (SIQ-Jr; Reynolds \& Mazza, 1999) is a 15-item self-report questionnaire that assesses the frequency and severity of SI and the measure demonstrated high reliability $(\alpha=0.96)$ within this sample. Participants rated the frequency of suicidal thoughts (e.g., "I wished I were dead," "I thought about what to write in a suicide note") on a 6-point Likert scale from 0 ("I never had this thought") to 6 ("Almost every day").

\section{COVID-19 Stressors}

A list of COVID-19 stressors was developed based on prior literature on stress in psychiatric adolescent samples (e.g., Rudolph \& Hammen, 1999). Youth rated the degree to which they experienced stressors on a 5-point Likert scale, from 1 (Not at all) to 5 (Extremely). The total stressor score (i.e., sum of items) was used $(\alpha=0.79)$ as a measure of total COVID-19 related stress. Our COVID-19 related measures are included as supplementary material (Fig. 2).

\section{COVID-19 Coping Strategies}

COVID-19-related coping strategies were assessed by the following question: "Before coming to the hospital, how much were you doing the following things to cope with COVID-19 (coronavirus) and the changes and rules put in place as a result of the virus?" Participants rated the frequency with which they had engaged in a list of strategies to cope with COVID-19 stress on a 5-point Likert scale from 1 (Not at all) to 5 (Very Often). This measure was derived based on coping theory suggesting individuals use both engagement and disengagement strategies (Compas et al., 2017) and adapted from prior measures used in adolescents (e.g., Measure of Adolescent Coping Strategies; Sveinbjornsdottir \& Thorsteinsson, 2014). Coping strategies were categorized as: social engagement (4 items; e.g., "Spending time with friends in person"; $\alpha=0.75$ ), effortful distraction ( 3 items; e.g., "Browsing the Internet/social media"; $\alpha=0.65$ ), physical activity (2 items; e.g., "Yoga"; $\alpha=0.49$ ), relaxation (1 item; "Using mindfulness, meditation, or relaxation strategies"), and avoidance or disengagement (4 items; e.g., "Avoiding thinking about what is going on"; $\alpha=0.70$ ). Due to low reliability, the physical activity coping scale was not used in analyses.

Fig. 2 Youth who reported negative COVID-19-related impact on their daily functioning evidenced higher levels of suicidal ideation at the time of admission $(n=103),{ }^{*} p<0.05$

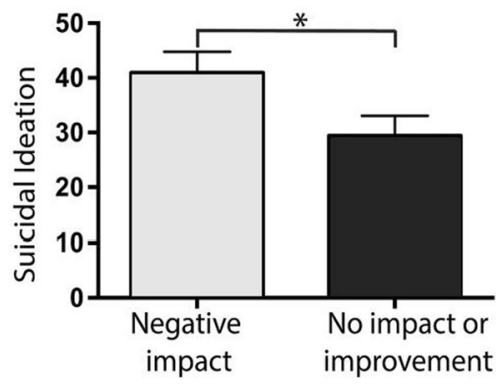




\section{COVID-19-Related Change in Functioning}

Perceived change in 12 domains of functioning (e.g., sleep, peer relationships, school) due to COVID-19 was assessed with the question: "How has COVID-19 (coronavirus) changed your day-to-day life?" Youth were categorized as having: (1) reported either no change or improved functioning on the majority of domains (coded 1); or (2) worsened functioning on the majority of domains (coded 0$)$.

\section{Coping Repertoire}

Responses were rated on a Likert scale from 1 (Not at all true) to 7 (Totally true). We administered the 6-item Multiple Coping Strategy Use scale of the Self-Perceived Flexible Coping with Stress Scale to assess adolescents' repertoire of coping skills (Zimmer-Gembeck et al., 2018). The items assessed whether adolescents report having multiple skills to draw from when faced with a stressor (e.g., "I can easily find new ways of coping with stress if needed." "Even when the stress is new to me, I can come up with a way to deal with it."). Responses were rated on a Likert scale from 1 (Not at all true) to 7 (Totally true). The scale showed excellent internal consistency in this sample $(\alpha=0.97)$.

\section{Clinical Symptoms}

The Pediatric Symptom Checklist-17 (PSC-17) was used to assess internalizing and externalizing symptoms and attention difficulties (Gardner et al., 1999). Youth are instructed to rate how well each of the behaviors (e.g., "Daydream too much") describes them. The response options are as follows: "Never" $=0$, "Sometimes" $=1$, "Often" $=2$. The measure demonstrated good internal consistency overall $(\alpha=0.81)$ in this sample. The internal consistency for each subscale ranged from satisfactory to good (attention difficulties subscale $\alpha=0.67$; externalizing symptoms subscale $\alpha=0.69$; internalizing symptoms subscale $\alpha=0.89$ ).

\section{Analytical Plan}

We first examined whether participant demographic characteristics, including age, gender identity (cisgender youth $=0$, vs. gender minority $=1$ ), and race/ethnicity (white non-Hispanic $=0$, vs. youth of color $=1$ ) were related to SI via correlations and general linear models. We provide descriptive data on youths' experiences of COVID-19 related stress, coping, and COVID-19-related impact on daily functioning. We also provided bivariate correlations among all study variables. We conducted two separate linear regression series to examine the associations between 1) COVID-19 stress related variables (i.e., COVID-19 related change in functioning and general level of COVID-19 related stress) and SI, and 2) coping repertoire and strategies (i.e. Social Engagement, Distraction, Relaxation, and Disengagement/Avoidance) with SI. 


\section{Results}

\section{Preliminary Analyses: Associations Among Demographic Variables and SI.}

Demographic variables were not significantly associated with SI (lowest $p>0.05$ ), and therefore were not included as covariates in our analyses.

\section{Characterization of COVID-19-Related Experience}

Overall, a large proportion of youth reported that the pandemic negatively impacted their daily functioning $(43.9 \% ; n=47)$. A comparable proportion of participants reported no overall COVID-19-related changes in daily functioning $(45.8 \% ; n=49)$. Notably, a relatively small proportion reported being mostly positively impacted by the COVID-19 pandemic $(6.5 \% ; n=7)$; these youths were combined with youth who reported no daily functioning impact in analyses. Four youth (3.7\%) noted an equal number of domains in which daily functioning was negatively, positively, or not impacted, and thus were excluded from all subsequent daily functioning analyses (daily functioning analysis $n=103$ ). Mean levels of COVID-19-related stress and rates of endorsement of each specific stressor are presented in Table 1. The three most frequently endorsed stressors were not being able to leave home when they want to, being cut off from contact with others, and being worried that someone they cared about may get sick with COVID-19. The two most frequently endorsed coping strategies in this sample were avoidance or disengagement and distraction.

In addition, youth reported mean levels of SI above the clinical cutoff score of 31 at the time of admission (see Table 2). Bivariate correlations among all study variables are also reported in Table 2 .

\section{COVID-19-Related Stress and Changes in Daily Functioning and SI}

We conducted a stepwise linear regression analysis to examine the associations of COVID-19-related impact on functioning prior to hospitalization and COVID-19-related

Table 1 Mean and standard deviation scores and rate of endorsement of COVID-19-related stressors

\begin{tabular}{lll}
\hline Stressor & M (SD) & $\begin{array}{l}\mathrm{n}(\%) \text { who endorsed the } \\
\text { stressor (Total } N=107)\end{array}$ \\
\hline Basic needs not being met & $1.52(0.93)$ & $33(30.84 \%)$ \\
Money problems & $1.70(1.06)$ & $40(37.38 \%)$ \\
Conflict at home & $2.33(1.32)$ & $67(62.62 \%)$ \\
Being cut off from contact with others & $2.55(1.40)$ & $70(65.42 \%)$ \\
Not being able to go out/leave the home & $2.89(1.29)$ & $85(79.44 \%)$ \\
Not being able to attend special events (e.g., graduation) & $2.60(1.43)$ & $71(66.36 \%)$ \\
Having to change who I'm living with & $1.50(0.94)$ & $28(26.17 \%)$ \\
Not being able to see people I care about in person & $2.89(1.37)$ & $80(74.77 \%)$ \\
Someone I care about got sick & $1.64(1.08)$ & $33(30.84 \%)$ \\
Worried about getting COVID-19 & $2.21(1.36)$ & $57(53.27 \%)$ \\
Worried about someone I care about getting COVID-19 & $2.80(1.39)$ & $80(74.77 \%)$ \\
\hline
\end{tabular}


Table 2 Means, standard deviations, and bivariate correlations between all variables

\begin{tabular}{|c|c|c|c|c|c|c|c|c|c|}
\hline & $\begin{array}{l}\text { Mean } \\
\text { (SD) }\end{array}$ & 1 & 2 & 3 & 4 & 5 & 6 & 7 & 8 \\
\hline \multicolumn{10}{|l|}{1} \\
\hline Suicidal & 34.59 & 1 & & & & & & & \\
\hline Ideation & (27.19) & & & & & & & & \\
\hline \multicolumn{10}{|l|}{2} \\
\hline COVID19 & 24.64 & 0.05 & 1 & & & & & & \\
\hline Stress & $(7.76)$ & & & & & & & & \\
\hline \multicolumn{10}{|l|}{3} \\
\hline Coping & 24.31 & $-0.49 * *$ & 0.13 & 1 & & & & & \\
\hline Repertoire & $(10.6$ & & & & & & & & \\
\hline \multicolumn{10}{|l|}{4} \\
\hline Social & 10.9 & -0.11 & 0.18 & $0.35 * *$ & 1 & & & & \\
\hline Engagement & $(3.86)$ & & & & & & & & \\
\hline \multicolumn{10}{|l|}{5} \\
\hline Distraction & 10.23 & 0.10 & $0.29 * *$ & 0.10 & $0.57 * *$ & 1 & & & \\
\hline & $(2.87)$ & & & & & & & & \\
\hline \multicolumn{10}{|l|}{6} \\
\hline Relaxation & 2.32 & -0.07 & 0.17 & $0.28 * *$ & $0.33 * *$ & $0.30 * *$ & 1 & & \\
\hline & $(1.27)$ & & & & & & & & \\
\hline \multicolumn{10}{|l|}{7} \\
\hline Disengagement/ & 12.3 & $0.22 *$ & $0.36 * *$ & -0.08 & $0.26 * *$ & $0.59 * *$ & 0.12 & 1 & \\
\hline Avoidance & $(3.61)$ & & & & & & & & \\
\hline \multicolumn{10}{|l|}{8} \\
\hline Change in & 12.3 & $-0.21 *$ & -0.09 & 0.15 & 0.19 & -0.01 & 0.08 & -0.08 & 1 \\
\hline Functioning & $(3.61)$ & & & & & & & & \\
\hline
\end{tabular}

${ }^{*} p<.05 ; * * p \leq .01$

COVID-19-related coping strategies were assessed by the following question: "Before coming to the hospital, how much were you doing the following things to cope with COVID-19 (coronavirus) and the changes and rules put in place as a result of the virus?" COVID-19-related stress was assessed by adding up all adolescents' ratings from a list of individual stressors

stress and SI. We found that COVID-19-related decrease in functioning was associated with greater SI when used as a single predictor in the model or when the levels of COVID-19-related stress were added to the model $(\beta=-11.56$, CI [-0.99- -22.14], $p=0.32 ; \beta=-11.30$, CI $[-0.65--21.96], p=0.38)$. COVID-19-related stress was not significantly associated with SI $(\beta=-11.56$, CI [-0.99- -22.14], $p=0.32)$ and did not significantly increase the amount of variability explained by the model $\left(\mathrm{R}^{2}=0.05, \mathrm{~F}\right.$ $(1,102)=4.71, p=0.03$ vs. $\left.\mathrm{R}^{2}=0.05, \mathrm{~F}(2,102)=2.48, p=0.09 ; \Delta \mathrm{R}^{2}<0.01, p=0.59\right)$. Thus, we used the generalized linear model with COVID-19-related impact on functioning as a sole predictor to depict the findings that among youth who reported decreased functioning in the context of COVID-19, SI was significantly higher $(M=40.94$, SEM $=3.83$ ), compared to those who reported no changes or improved functioning $(\mathrm{M}=29.38, \mathrm{SEM}=3.67)$ (Fig. 2). 


\section{Coping Strategies and Repertoire in Relation to SI}

Next, we conducted a series of linear regression analyses using coping repertoire and coping strategies (i.e., Social Engagement, Distraction, Relaxation, and Disengagement/Avoidance) as predictors and SI as a criterion variable. Coping repertoire was the only significant predictor of SI $(\beta=-1.23$, CI $[-0.76--1.70], p<0.001)$, whereas neither Social Engagement $(\beta=-0.29$, CI $[-1.84-1.26], p=0.71)$, Distraction $(\beta=0.96$, CI $[-1.38-3.30]$, $p=0.42)$, Relaxation $(\beta=0.99$, CI $[-2.92-4.90], p=0.62)$, or Disengagement/Avoidance $(\beta=0.83$, CI $[-0.76-2.43], p=0.30)$ were significantly associated with SI in the model that included all of these variables as predictors. Notably, the link between coping repertoire and SI was significant when it was a sole predictor as well as with all other coping strategies in the model (all ps $<0.05$ ). These results highlight the role of a wider coping repertoire over the use of specific coping strategies in SI in adolescents who are at an increased risk for suicide.

\section{Discussion}

The current study sought to characterize the experience of psychiatrically hospitalized youth during the COVID-19 pandemic. Specifically, we examined COVID-19-related clinical indices of risk, including stress, coping strategy use and repertoire, and functioning, as they relate to SI in a high-risk sample. With regard to characterizing the experiences of these youth during the pandemic, results showed nearly half of the sample reported COVID-19 negatively impacted daily functioning, and very few youth indicated COVID19 positively impacted them with regard to daily functioning. These findings indicate the clear impact of the pandemic on adolescents vulnerable to serious mental health problems, and suggest more research is needed to understand both risk and resilience in this population.

Further, youth most commonly reported not being able to leave home when they want to, being cut off from contact with others, and being worried that someone they cared about may get sick with COVID-19 as key stressors during the pandemic. Interpersonal stressors such as social isolation may be especially salient for adolescents, as this developmental period is characterized by a greater reliance on peers for support and socialization. Prior research also indicates social isolation is associated with suicidal outcomes (Calati et al., 2019), highlighting the importance of assessing these types of stressors in youth during the pandemic. To cope with these stressors, youth primarily endorsed the use of avoidance, a disengagement coping strategy, and distraction, an engagement strategy.

SI ratings, which were on average above the clinical cutoff in this high-risk sample, were significantly higher among youth who reported worsening in functioning, compared to those who reported positive or no changes in functioning due to COVID-19. SI ratings were also elevated among youth who had fewer coping strategies at their disposal. These findings indicate the relevance of COVID-19-related stress for psychological functioning among youth at high risk for suicide.

Prior research indicates the use of engagement coping strategies can be a protective factor for suicide, whereas disengaged coping may increase suicide risk (Mirkovic et al., 2020; Nrugham et al., 2012). Along those lines, although higher levels of COVID-related stress were not linked to greater SI, they were associated with the use of disengagement 
strategies, rather than more engagement-focused coping strategies (e.g., social engagement or relaxation). Disengagement coping was also associated with higher levels of SI, which is consistent with prior research in adolescents (Horwitz et al., 2011). This finding is also consistent with the broader literature on emotion regulation and SI, which indicates overall difficulties in regulation are associated with elevated symptoms (Miranda et al. 2012).

Contrary to our hypothesis, there was no significant relation between greater use of social engagement, distraction, and relaxation coping and SI. Horowitz and colleagues examined associations among coping strategy use and SI among youth presenting to the emergency department, and found a similar pattern of bivariate results, such that maladaptive strategies (e.g., disengagement, self-blame, denial) were associated with SI severity but adaptive strategies were not (with the exception of emotional support) (Horowitz, Hill, $\&$ King, 2011). Further research examining the full spectrum of coping strategies that adolescents may engage in to manage stress, including stress specific to COVID-19, is needed to clarify the role of engagement-focused strategies in SI risk. It is possible that strategies typically considered adaptive, such as social engagement, may function qualitatively differently in the context of COVID-19. For example, a sudden shift to engaging solely online, with very limited in-person social engagement during the early phases of the pandemic, could influence how social engagement operates as a coping strategy.

Furthermore, in multivariate analyses, coping repertoire, rather than specific strategy use, was predictive of SI severity. The finding that having access to a larger repertoire of coping strategies was linked to lower SI is in line with previous theoretical and empirical research that highlighted the advantages of flexible coping across multiple stressors, and extends it to coping with COVID-19-related stress among at-risk youth (Cheng, 2001; Bonanno \& Burton, 2013;; Gross, 2015; Southward et al., 2018). Given the challenges and required adaptations COVID-19 presents for youth in completing daily activities, adolescents may benefit from assistance in adapting and broadening their preferred coping strategies and focusing on what they can control, to prevent experiencing helplessness or avoidance.

\section{Limitations and Future Directions.}

The cross-sectional study design restricts our ability to draw causal or temporal conclusions regarding the constructs examined. Longitudinal research examining the impact of COVID-19 following psychiatric hospitalization is needed to better understand how these processes may impact risk for SI over time. Further, we were limited to a single informant (youth); research examining parents' perceptions of youths' experiences of stress, coping, and their mental health in the pandemic would be a valuable next step in this research. The level of government-mandated restrictions has also varied over the timeframe of data collection, and as such, the date of assessment may have impacted responses to the pandemic. An important study limitation is the reliance on new measures, developed in response to the COVID-19 pandemic, as well as the use of single-item measures to assess some of our key study constructs. At the time of study design, validated measures were not yet available to assess COVID-19-related functioning, stressors, nor coping. Thus, measures were developed for this study and have not been formally psychometrically validated. Further, the sample size and number of items precludes us from conducting a factor analysis to explore whether the engagement and disengagement strategy distinction holds in this measure. As the pandemic has continued, it will be important for future work to establish validated and reliable instruments to assess pandemic-related experiences. 
Finally, data shows Black and Indigenous communities are disproportionately impacted by the pandemic, and research is needed to understand how coping and experiences of stress related to COVID-19 may differ in Black and Indigenous adolescents (APM Research Lab, 2020). In this study, we were unable to examine the specific experiences of these groups during the COVID-19 pandemic, due to limitations in sample size and the setting from which this sample was drawn. Similarly, the small number of gender minority youth in our sample precluded us from examining the impact of COVID-19 on mental health outcomes in these youth. Given well-documented mental health disparities experienced by gender minority youth (Bettis et al., 2020; Delozier et al., 2020; Thoma et al., 2019), targeted research focusing on the experiences of gender minority youth during the COVID-19 pandemic is needed.

\section{Clinical Implications}

Findings underscore the importance of assessing pandemic-specific distress among psychiatrically hospitalized adolescents. Youth reporting a decline in functioning may be at particularly high risk for SI and ineffective coping. Enriching adolescents' coping strategies repertoire and helping them develop specific, engagement-focused coping plans may be critical to facilitating the health and well-being of at-risk youth during this challenging and uncertain time. Further, supporting youth in reducing their reliance on disengagement-focused strategies may be especially important for SI risk. Importantly, identifying skills that are readily accessible within the constraints of COVID-19-related safety measures is important.

\section{Conclusion}

The COVID-19 pandemic has radically transformed the lives of adolescents in the U.S. In this study, the association of COVID-19-related stress and coping strategies and repertoire with SI were examined in a sample of adolescent inpatients. For adolescents vulnerable to SI, findings demonstrate that limited coping repertoire and effectiveness, the use of disengagement coping strategies, and COVID-19-related disruptions in daily functioning were associated with greater SI.

Supplementary Information The online version contains supplementary material available at https://doi. org/10.1007/s10566-021-09641-1.

Funding This work was supported by the National Institutes of Mental Health [Kudinova: K23MH122587; Bettis: K23122737; Thompson: K23MH119211; Burke: T32 MH019927; Nesi: K23MH122669]. Jacqueline Nesi is also supported by grant PDF-010517 from the American Foundation for Suicide Prevention. Sarah A. Thomas is partially supported by Institutional Development Award Number U54GM115677 from the National Institute of General Medical Sciences of the National Institutes of Health, which funds Advance Clinical and Translational Research (Advance-CTR). NIH and AFSP had no role in the design and conduct of the study; collection, management, analysis, and interpretation of the data; and preparation, review, or approval of the manuscript; or decision to submit the manuscript for publication. The content is solely the responsibility of the authors. 


\section{References}

APM Research Lab (2020, August 19). The color of coronavirus: COVID-19 deaths by race and ethnicity in the U.S. https://www.apmresearchlab.org/covid/deaths-by-race

Asmundson, G. J., Paluszek, M. M., Landry, C. A., Rachor, G. S., McKay, D., \& Taylor, S. (2020). Do pre-existing anxiety-related and mood disorders differentially impact COVID-19 stress responses and coping? Journal of Anxiety Disorders, 74, 102271.

Bettis, A. H., Thompson, E. C., Burke, T. A., Nesi, J., Kudinova, A. Y., Hunt, J. I., Liu, R. T., \& Wolff, J. C. (2020). Prevalence and clinical indices of risk for sexual and gender minority youth in an adolescent inpatient sample. Journal of Psychiatric Research, 130, 327-332.

Bonanno, G. A., \& Burton, C. L. (2013). Regulatory flexibility: An individual differences perspective on coping and emotion regulation. Perspectives on Psychological Science: A Journal of the Association for Psychological Science, 8, 591-612, doi: 10.1177/1745691613504116

Calati, R., Ferrari, C., Brittner, M., Oasi, O., Olié, E., Carvalho, A. F., \& Courtet, P. (2019). Suicidal thoughts and behaviors and social isolation: A narrative review of the literature. Journal of Affective Disorders, 245, 653-667, doi: 10.1016/j.jad.2018.11.022

Cheng, C. (2001). Assessing coping flexibility in real-life and laboratory settings: A multimethod approach. Journal of Personality and Social Psychology, 80, 814-833, doi: 10.1037//0022-3514.80.5.814

Compas, B., Connor-Smith, J., Saltzman, H., Thomsen, A., \& Wadsworth, M. (2001). Coping with stress during childhood and adolescence: Problems, progress, and potential in theory and research. Psychological Bulletin, 127, 87-127, doi: 10.1037/0033-2909.127.1.87

Compas, B. E., Jaser, S. S., Dunn, M. J., \& Rodriguez, E. M. (2012). Coping with chronic illness in childhood and adolescence. Annual Review of Clinical Psychology, 8, 455-480, doi: 10.1146/ annurev-clinpsy-032511-143108

Compas, B. E., Jaser, S. S., Bettis, A. H., Watson, K. H., Gruhn, M. A., Dunbar, J. P., \& Thigpen, J. C. (2017). Coping, emotion regulation, and psychopathology in childhood and adolescence: A meta-analysis and narrative review. Psychological Bulletin, 143(9), 939.

Danese, A., \& McEwen, B. S. (2012). Adverse childhood experiences, allostasis, allostatic load, and agerelated disease. Physiology \& Behavior, 106, 29-39. https://doi.org/10.1016/j.physbeh.2011.08.019

Delozier, A. M., Kamody, R. C., Rodgers, S., \& Chen, D. (2020). Health disparities in transgender and gender expansive adolescents: A topical review from a minority stress framework. Journal of Pediatric Psychology, 45(8), 842-847.

Duan, L., Shao, X., Wang, Y., Huang, Y., Miao, J., Yang, X., \& Zhu, G. (2020). An investigation of mental health status of children and adolescents in china during the outbreak of COVID-19. Journal of Affective Disorders, 275, 112-118. https://doi.org/10.1016/j.jad.2020.06.029

Ellis, W. E., Dumas, T. M., \& Forbes, L. M. (2020). Physically isolated but socially connected: Psychological adjustment and stress among adolescents during the initial COVID-19 crisis. Canadian Journal of Behavioural Science/revue Canadienne Des Sciences Du Comportement, 52(3), 177.

Fegert, J. M., Vitiello, B., Plener, P. L., \& Clemens, V. (2020). Challenges and burden of the Coronavirus 2019 (COVID-19) pandemic for child and adolescent mental health: A narrative review to highlight clinical and research needs in the acute phase and the long return to normality. Child and Adolescent Psychiatry and Mental Health, 14, 1-11.

Golberstein, E., Wen, H., \& Miller, B. F. (2020). Coronavirus disease 2019 (COVID-19) and mental health for children and adolescents. JAMA Pediatrics, 174, 819.

Golberstein, E., Gonzales, G., \& Meara, E. (2019). How do economic downturns affect the mental health of children? evidence from the National Health Interview Survey. Health Economics, 28(8), 955-970.

Gross, J. J. (2015). Emotion regulation: Current status and future prospects. In Psychological inquiry (Vol. 26, Issue 1, pp. 1-26). Taylor Francis. https://doi/org/10.1080/1047840X.2014.940781

Gruber, J., Prinstein, M. J., Clark, L. A., Rottenberg, J., Abramowitz, J. S., Albano, A. M., \& Forbes, E. E. (2020). Mental health and clinical psychological science in the time of COVID-19: Challenges, opportunities, and a call to action. American Psychologist, 76(3), 409-426.

Guessoum, S. B., Lachal, J., Radjack, R., Carretier, E., Minassian, S., Benoit, L., \& Moro, M. R. (2020). Adolescent psychiatric disorders during the COVID-19 pandemic and lockdown. Psychiatry Research, 291, 113264. https://doi.org/10.1016/j.psychres.2020.113264

Gunnell, D., Appleby, L., Arensman, E., Hawton, K., John, A., Kapur, N., \& Chan, L. F. (2020). Suicide risk and prevention during the COVID-19 pandemic. The Lancet Psychiatry, 7(6), 468-471.

Hammen, C. (2005). Stress and depression. Annual Review of Clinical Psychology, 1, 293-319.

Holmes, E. A., O’Connor, R. C., Perry, V. H., Tracey, I., Wessely, S., Arseneault, L., \& Ford, T. (2020). Multidisciplinary research priorities for the COVID-19 pandemic: a call for action for mental health science. The Lancet Psychiatry, 7(6), 547-560. 
Horwitz, A. G., Hill, R. M., \& King, C. A. (2011). Specific coping behaviors in relation to adolescent depression and suicidal ideation. Journal of Adolescence, 34(5), 1077-1085.

Jiao, W. Y., Wang, L. N., Liu, J., Fang, S. F., Jiao, F. Y., Pettoello-Mantovani, M., \& Somekh, E. (2020). Behavioral and emotional disorders in children during the COVID-19 epidemic. The Journal of Pediatrics, 221, 264-266.e1. https://doi.org/10.1016/j.jpeds.2020.03.013

Liu, R. T., \& Miller, I. (2014). Life events and suicidal ideation and behavior: A systematic review. Clinical Psychology Review, 34(3), 181-192.

Miranda, R., Gallagher, M., Bauchner, B., Vaysman, R., \& Marroquín, B. (2012). Cognitive inflexibility as a prospective predictor of suicidal ideation among young adults with a suicide attempt history. Depression and Anxiety, 29, 180-186. https://doi.org/10.1002/da.20915

Mirkovic, B., Cohen, D., de La Rivière, S. G., Pellerin, H., Guilé, J. M., Consoli, A., \& Gerardin, P. (2020). Repeating a suicide attempt during adolescence: risk and protective factors 12 months after hospitalization. European child \& adolescent psychiatry. https://doi.org/10.1007/s00787-020-01491-x

Nrugham, L., Holen, A., \& Sund, A. M. (2012). Suicide attempters and repeaters: Depression and copinga prospective study of early adolescents followed up as young adults. The Journal of Nervous and Mental Disease, 200(3), 197-203.

O’Connor, R. C., Rasmussen, S., \& Hawton, K. (2010). Predicting depression, anxiety and self-harm in adolescents: The role of perfectionism and acute life stress. Behaviour Research and Therapy, 48(1), 52-59.

Polizzi, C., Lynn, S. J., \& Perry, A. (2020). Stress and coping in the time of COVID-19: Pathways to resilience and recovery. Clinical Neuropsychiatry, 17(2), 59-62.

Reynolds, W. M., \& Mazza, J. J. (1999). Assessment of suicidal ideation in inner-city children and young adolescents: Reliability and validity of the suicidal ideation questionnaire-JR. School Psychology Review, 28(1), 17-30.

Rudolph, K. D., \& Hammen, C. (1999). Age and gender as determinants of stress exposure, generation, and reactions in youngsters: A transactional perspective. Child Development, 70(3), 660-677.

Singh, S., Roy, D., Sinha, K., Parveen, S., Sharma, G., Joshi, G. (2020). Impact of COVID-19 and lockdown on mental health of children and adolescents: A narrative review with recommendations. Psychiatry Research, 293, 113429. https://doi.org/10.1016/j.psychres.2020.113429

Southward, M. W., Altenburger, E. M., Moss, S. A., Cregg, D. R., \& Cheavens, J. S. (2018). Flexible, yet firm: A model of healthy emotion regulation. Journal of Social and Clinical Psychology, 37, 231-251. https://doi.org/10.1521/jscp.2018.37.4.231

Sveinbjornsdottir, S., \& Thorsteinsson, E. B. (2014). Psychometric properties of the Measure of Adolescent Coping Strategies (MACS). Psychology, 5, 142-147. https://doi.org/10.4236/psych.2014.52022

Thoma, B. C., Salk, R. H., Choukas-Bradley, S., Goldstein, T. R., Levine, M. D., \& Marshal, M. P. (2019). Suicidality disparities between transgender and cisgender adolescents. Pediatrics. https://doi.org/10. 1542/peds.2019-1183

Thompson, E. C., Thomas, S. A., Burke, T. A., Nesi, J., MacPherson, H. A., Bettis, A. H., Kudinova, A. Y., Affleck, K., Hunt, J., \& Wolff, J. C. (2021). Suicidal thoughts and behaviors in psychiatrically hospitalized adolescents pre- and post- COVID-19: A historical chart review and examination of contextual correlates. Journal of Affective Disorders Reports, 4, 100100. https://doi.org/10.1016/j.jadr.2021. 100100

Zimmer-Gembeck, M. J., Skinner, E. A., Modecki, K. L., Webb, H. J., Gardner, A. A., Hawes, T., \& Rapee, R. M. (2018). The selfperceptionof flexible coping with stress: A new measure and relations with emotional adjustment. In Cogent psychology (Vol. 5, Issue 1).Taylor \& Francis. https://doi.org/10.1080/ 23311908.2018.1537908

Publisher's Note Springer Nature remains neutral with regard to jurisdictional claims in published maps and institutional affiliations. 


\section{Authors and Affiliations}

Anastacia Y. Kudinova ${ }^{1,2}$ - Alexandra H. Bettis ${ }^{3}$. Elizabeth C. Thompson ${ }^{1,4}$. Sarah A. Thomas $\mathrm{s}^{1,4} \cdot$ Jacqueline Nesi $^{1,4} \cdot$ Leyla Erguder $^{1,2} \cdot$ Heather A. MacPherson ${ }^{1,2}$. Taylor A. Burke ${ }^{1,4}$. Jennifer C. Wolff ${ }^{1,2}$

1 Warren Alpert Medical School of Brown University, Providence, RI, USA

2 Bradley Hospital, East Providence, Rhode Island, USA

3 Vanderbilt University Medical Center, Nashville, TN, USA

4 Rhode Island Hospital, East Providence, RI, USA 